Formación Universitaria

Vol. 7(2), 41-50 (2014)

doi: $10.4067 / S 0718-50062014000200006$

\title{
Taller Multidisciplinario para el Desarrollo de Competencias de Comunicación Lingüística de la Investigación
}

\author{
María del S. Marines, Norma G. Heredia, Luis E. Solís, Daniel A. Mena \\ Universidad Autónoma de Yucatán, Facultad de Ingeniería, Av. Industrias no Contaminantes x Anillo \\ Periférico Norte s/n Apartado Postal No. 150 Cordemex CP 97310, Mérida, Yucatán-México \\ (e-mail: marinesg@uady.mx)
}

Recibido Nov. 13, 2013; Aceptado Dic. 31, 2013; Versión final recibida Ene. 15, 2014

\begin{abstract}
Resumen
Se describe el diseño y evaluación de un taller multidisciplinario, enfocado a promover la adquisición de competencias de comunicación lingüística y de investigación en los alumnos que cursan licenciatura de ingeniería en la Universidad Autónoma de Yucatán para reforzar su formación integral. El taller está enfocado para ayudar a los alumnos a expresarse de una mejor manera en equipos de aprendizaje cooperativo multidisciplinarios. La implementación del taller implicó la coordinación académica entre profesores, quienes se involucraron de acuerdo a las asignaturas que imparten, realizando evaluaciones integrales en las que cada profesor calificó el nivel de competencia alcanzado. Los alumnos participantes, fueron evaluados antes y después del taller. A partir de los resultados obtenidos, se concluyó que el taller demostró su efectividad para elevar el nivel de competencias de comunicación lingüística y de investigación de los alumnos participantes.
\end{abstract}

Palabras clave: competencias, taller multidisciplinario, formación integral, equipos de aprendizaje cooperativo, comunicación lingüística.

\section{Taller Multidisciplinario para el Desarrollo de Competencias de Comunicación Lingüística de la Investigación}

\begin{abstract}
The design and evaluation of a multidisciplinary workshop, focused on promoting the acquisition of linguistic communication and research skills in undergraduate engineering students of the Autonomous University of Yucatán in Mexico to strengthen their comprehensive training. The workshop is aimed to help students to express themselves in a better way in multidisciplinary cooperative learning teams. The implementation of the workshop involved the academic coordination between teachers who were involved according to the subjects they teach, conducting comprehensive assessments that each teacher evaluated the level of competence achieved. Participating students were evaluated before and after the workshop. From the results, it was concluded that the workshop proved to be very effective in raising the level of skills of linguistic communication and research of participating students.
\end{abstract}

Keywords: skills, multidisciplinary workshop, comprehensive training, cooperative learning teams, linguistic communication 


\section{INTRODUCCIÓN}

La comunicación es un proceso complejo en el que inciden factores personales, culturales, contextuales y circunstanciales. En los últimos años, a raíz de las tendencias nacionales e internacionales de la educación superior, se ha hecho muy evidente la existencia de barreras de comunicación que de una u otra forma impiden obtener resultados académicos satisfactorios; estas barreras que se van acentuando conforme se van interrelacionando las diversas disciplinas para la resolución de problemas en el ámbito profesional, contribuyen a hacer más difícil la toma de decisiones. En este contexto, la toma de decisiones se hace más difícil durante la interrelación de profesionales que poseen conocimientos especializados en cada disciplina formativa. Según estas recientes formas de interacción, existe un marcado contraste con respecto a los procesos educativos un tanto rígidos que intentan seguir con los modelos de enseñanza tradicional, conductista y hasta cierto punto con el modelo constructivista, siendo que este último ahora ya no es suficiente para el logro de la tan añorada verdadera formación integral, puesto que no representa una garantía plena para alcanzar los objetivos que hoy en día se plantean en los planes de estudio.

La enseñanza superior está dividida en áreas de conocimiento o disciplinas, todas ellas utilizan el lenguaje; en la transformación del conocimiento es donde se involucra al estudiante en distintas fases de comprensión y organización de fenómenos que se estudian, esto hace difícil la apropiación del conocimiento. Al respecto, Carlino (2003) señala que "la transformación del conocimiento, de partida ocurre, sólo cuando el que escribe tiene en cuenta las necesidades informativas de su potencial lector y desarrolla un proceso dialéctico entre el conocimiento previo y las exigencias retóricas para producir un texto adecuado".

Si se plantea un cambio significativo en los egresados para que éstos se inserten en la sociedad de manera responsable y eficiente, hay que tener en cuenta que debe hacerse algo para que desaparezcan las barreras de comunicación inter y multidisciplinarias. Es muy notorio que los egresados presentan diversos niveles de carencias de capacidades para comunicarse adecuadamente y sobre todo para redactar documentos de carácter científico; no existen en los actuales planes de estudio, asignaturas o talleres que fomenten la lectura, comprensión y redacción científica, ni tampoco se cuenta con metodologías especializadas que coadyuven a la adquisición de competencias para que los futuros egresados desarrollen una buena práctica de la comunicación del conocimiento. Aunque, si bien es cierto que las Instituciones de Educación Superior, entre las que se encuentra la Universidad Autónoma de Yucatán, se han preocupado en los años recientes por considerar los aspectos más especializados para una mejor formación, ésta se ha centrado prácticamente en los aspectos de vocación profesional, capacidad académica, recursos tecnológicos, todo ello con el noble propósito de abatir el rezago educativo y la deserción escolar, así como el de incrementar los índices de aprovechamiento académico y de eficiencia terminal, flexibilizando los currículos y consolidando una oferta educativa de calidad. También es cierto que deben atenderse las necesidades detectadas de comunicación con un lenguaje eficaz y eficiente para el logro de las relaciones inter y multidisciplinarias a nivel nacional e internacional. Se ha escrito mucho acerca de la fundamentación de los componentes, de las habilidades de comunicación y de metodologías para la investigación, pero muy poco se ha estudiado acerca de su inserción transversal en los planes de estudio enfocados a las ingenierías. Castellanos (2003) realizó un estudio cualitativo utilizando una guía de observación estructurada que tomaba en consideración ciertos indicadores de la competencia investigativa, para observar de manera sistemática la actividad de ciento cincuenta estudiantes de la educación superior en la Ciudad de la Habana. Estos indicadores fueron: la posibilidad de seguir una línea propia de pensamiento y procesamiento autónomos, poseer una variedad de ideas y opciones inusuales que el sujeto puede ofrecer ante un problema o situación, tener la opción de modificar el rumbo de la actividad intelectual cuando la situación lo requiere, mostrar economía de recursos como la relación entre el ritmo de procesamiento de la información y ejecución; así como la adecuación y calidad que se va logrando en dicho procesamiento. Los resultados encontrados llevaron a Castellanos (2003) a la conclusión de que las habilidades investigativas están integradas por tres componentes: el cognitivo, el metacognitivo, el motivacional y por ciertas cualidades de personalidad.

Polaco y Manjarrez (2010) sostienen que la multidisciplinariedad involucra el conocimiento de varias disciplinas, cada una de ellas aportando desde su espacio al tema en cuestión, lo anterior refiere a: la división de los campos científicos, al desarrollo y necesidades de las ramas del saber, a lo más específico y propio del desarrollo científico-técnico, a la profundización de los conocimientos y que es la posible integración de las diversas disciplinas en el proceso de investigación de un problema dado. La transdisciplinariedad, según Nicolescu (2002), implica comprender la realidad completa, con sus múltiples dimensiones, en contraste con fragmentos de un nivel de realidad que genera el trabajo disciplinar o interdisciplinario. Es decir, la comprensión del hoy mundo actual globalizado no se alcanza con los estudios disciplinares. La relación docentes/alumnos queda establecida en la realización de una tarea común: organizados cogestionariamente y autogestionariamente (Ander-Egg, 1991), en el que la transdisciplinariedad coadyuva hacia un conocimiento superior emergente, producto de un movimiento 
dialéctico de retro-alimentación y pro-alimentación del pensamiento, que nos permite cruzar las fronteras de diferentes áreas del conocimiento disciplinar y presentar imágenes de la realidad más integradas, en consecuencia, más ajustadas a las necesidades del momento (Martínez, 1999).

Las competencias se entienden como actuaciones integrales para identificar, interpretar, argumentar y resolver problemas del contexto con idoneidad y ética, integrando el saber ser, el saber hacer y el saber conocer (Tobón, Pimienta y García Fraile, 2010). Una competencia es el conjunto de conocimientos, habilidades y destrezas, tanto generales, como específicas y transversales, que debe tener un egresado con el fin de lograr un desempeño profesional exitoso; la adquisición de competencias impactan en las actitudes y valores del ser humano, y la tendencia actual es que los hace ser más responsables en su desempeño para con la sociedad y con el medio ambiente. Bellocchio (2010) describe las diez características que revisten las competencias de entre las que se puede destacar la que permite al ser humano adaptarse a diferentes situaciones para responder exitosamente en escenarios complejos y novedosos.

Tobón (2008) considera que la Educación Basada en Competencias (EBC) puede tener los siguientes modelos epistemológicos (Bellocchio, 2010, pp. 27-28): funcionalista (identificación de funciones a realizar para el logro de objetivos), conductual-organizacional (considera únicamente la conducta observable en el desarrollo de competencias), constructivista (importancia de conocimientos previos y construcción de competencias profesionales) y complejo (método hermenéutico de construcción del saber humano). Las investigaciones de Piaget (2005, 2006 y 2008) respecto del constructivismo, constituyen una importante aportación para explicar y comprender cómo se genera el conocimiento y el desarrollo psíquico del alumno, entendiendo al aprendizaje como un proceso de construcción activo, interactivo e individual, dando lugar a la formación sucesiva de estructuras mentales cada vez más complejas. La teoría de Ausubel (1990) ha ejercido una gran incidencia en la pedagogía contemporánea ya que ha defendido la idea de que los profesores tengan en cuenta los conocimientos previos, es decir, lo que los alumnos ya saben; criticó la enseñanza repetitiva tradicional proponiendo la necesidad de diseñar organizadores previos a partir de los cuales los alumnos puedan ser capaces de establecer relaciones significativas entre los conocimientos que poseen y los nuevos contenidos que estudian.

A través del concepto de competencia lingüística Chomsky, citado por Maldonado (2005), da cuenta de la manera como los seres humanos se apropian del lenguaje y lo emplean para comunicarse. La tesis central de dicho autor es que los seres humanos pueden producir y comprender nuevas oraciones, así como rechazar otras por no ser gramaticalmente correctas con base en su limitada experiencia lingüística. Estos planteamientos guardan ideas claves, un sistema de principios y reglas dispuestos en módulos, aprendizaje normado por reglas, relaciones entre competencia y desempeño, conocimiento implícito, posibilidades creativas a partir de normas, etc., aspectos retomados posteriormente por la psicolingüística y la psicología cognitiva. Esta relación con estudios lingüísticos acorta el camino a la pedagogía basada en competencias y conducirá a establecer la relación entre competencia y desempeño, donde se va a manifestar la competencia y alude a que la competencia siempre implica el desarrollo de posibilidades de desempeño en los sujetos (Ruiz, 2010). Hymes (1972) estableció el concepto de competencia comunicativa que toma en cuenta las actitudes, los valores y las motivaciones relacionadas con las lenguas, con sus características y sus usos; una persona competente en el lenguaje es aquella que lo emplea para integrarse con los otros, entendiendo y haciéndose entender. Las competencias en comunicación surgen como una salida al dilema siempre presente de la evaluación con respecto al lenguaje.

Para Vigotsky (1996), el lenguaje es el condicionante social fundamental del pensamiento humano, y propuso la idea de que las funciones psicológicas son el resultado de un proceso de apropiación de la cultura; postuló la Ley Genética del Desarrollo que afirma que toda función psíquica aparece en dos planos, en primera instancia, en el interpersonal y, posteriormente, se reconstruye internamente en el plano intrapersonal. En el marco de la teoría psicopedagógica, presenta una especial relevancia la categoría de zona de desarrollo próximo que Vigotsky (1994) la define como "No es otra cosa que la distancia entre el nivel de desarrollo real, determinado por la capacidad de resolver independientemente un problema, y el nivel de desarrollo potencial, determinado a través de la resolución de un problema bajo la guía de un adulto o en colaboración con otro compañero más capaz", es decir, que define una zona donde la acción del profesor incide de manera importante en el logro del aprendizaje significativo.

El aprendizaje basado en competencias actualmente es desarrollado y aplicado en México tal como muestran los trabajos de Regalado et al (2011) y Rodríguez et al (2011). Agudo et al (2013) han estudiado la percepción de adquisición de competencias transversales por parte de alumnos en una universidad pública en España. 
Canales y Schmal (2013) proponen como una forma de desarrollar la habilidad de comunicación escrita en una carrera universitaria de pregrado, la presentación de trabajos en forma de pósteres o paneles, como se usan en los congresos y conferencias. Así mismo, Benítez y García (2013) proponen el desarrollo de proyectos en el aula para recuperar el interés de los alumnos en la ciencia. Castro et al (2004), llevan a cabo la realización de una experiencia de adaptación de textos para su representación, partiendo de un texto narrativo (básicamente cuento) hasta su reelaboración como texto dramático y texto escénico para lograr el desarrollo de competencias de comunicación. Werner (1986) proponía y reflexionaba sobre la necesidad de realizar actividades en los cursos para mejorar las competencias de comunicación. Squier et al (2006) proponen la inclusión de un módulo de comunicación en un curso de laboratorio de química y más recientemente Mittendorf y Cox (2013) realizan una actividad de aprendizaje en un curso de bioquímica para mejorar las destrezas de comunicación basándose en el formato de un programa de televisión.

El Modelo Educativo para la Formación Integral (MEFI) (UADY, 2010), definido como respuesta a las tendencias mundiales de la educación superior, atiende a la Misión y Visión institucionales, dando cabal cumplimiento a uno de los quince programas prioritarios establecidos en el Plan Institucional de Desarrollo 2010-2020. El MEFI considera la formación integral de los alumnos como un proceso continuo tomando en cuenta las cinco dimensiones que lo conforman como ser humano: física, emocional, cognitiva, social, y valoral-actitudinal. Bajo este esquema y para orientar el quehacer educativo institucional, el MEFI contiene seis ejes fundamentales para la gestión educativa: Flexibilidad, Innovación, Internacionalización, Educación Basada en Competencias (EBC), Educación Centrada en el Aprendizaje (ECA), y Responsabilidad Social.

La búsqueda de soluciones profesionales asertivas que impacten positivamente en la resolución de los problemas de la sociedad, ha sido planteada como una acción de alta prioridad en la Universidad Autónoma de Yucatán (UADY). A la par con aquéllas, se visualizan las necesidades de comunicación lingüística y de investigación con eficacia y eficiencia que se pusieron de manifiesto a través del análisis de los resultados de una encuesta exploradora que se administró en el 2008 a cien alumnos de las licenciaturas en Ingeniería Civil, Ingeniería Física e Ingeniería en Mecatrónica, con el propósito de conocer la percepción de los alumnos respecto de los procesos de enseñanza y aprendizaje. El cuestionario reveló que los principales problemas percibidos por parte de los alumnos, en orden de importancia para ellos, eran: monotonía de los procesos curriculares, falta de motivación de los docentes hacia los estudiantes para la dedicación al aprendizaje, falta de confianza de los alumnos con relación a su aprendizaje, falta de compromiso de los docentes y el incumplimiento de los objetivos didácticos de los programas de asignatura. Todo ello llevó a la conclusión de que se requiere lograr cambios actitudinales en los actores del proceso enseñanzaaprendizaje, así como la aplicación de metodologías innovadoras de enseñanza, todo ello direccionado hacia la formación por competencias. El Campus de Ciencias Exactas e Ingenierías, en su Plan de Desarrollo 2010-2020, con el fin de lograr la formación integral de profesionales, considera ofrecer cursos y talleres para incrementar y potenciar las capacidades de comunicación oral y escrita, comprensión lectora y pensamiento lógico de los estudiantes, para lo cual se deben diseñar actividades de aprendizaje basadas en estudio de casos, aprendizaje basado en proyectos, utilización de escenarios reales, uso de simuladores y plataformas educativas, conjuntamente con el desarrollo y fortalecimiento de actividades de investigación.

\section{MATERIALES Y MÉTODOS}

Se realizó el diseño curricular del taller de acuerdo a la metodología de Bellocchio (2010), que consiste básicamente en un enfoque por competencias fundamentadas en el constructivismo, atendiendo los principios epistemológicos de la educación centrada en el estudiante, que permita el aprendizaje significativo, así como también la habilidad para seguir aprendiendo a lo largo de su vida. Se planteó como una propuesta para la adquisición de competencias, como un mecanismo de mejora educativa relacionada estrechamente con el perfil de egreso del estudiante de ingeniería considerando la dimensión social, el trabajo cooperativo, interacción en grupos homogéneos y heterogéneos en lo referente a las disciplinas diversas. El taller se centró en la adquisición de competencias de comunicación de conocimientos científicos, implicando necesariamente la coordinación académica entre profesores para la solución de problemas que involucraron asignaturas de diferentes niveles del plan de estudios, realizando evaluaciones integrales en las que cada profesor calificó el nivel de competencia alcanzado según su área. Para la validación de la propuesta se realizó un estudio previo para conocer la percepción de los estudiantes de ingeniería respecto de su proceso de enseñanza y aprendizaje; como resultado de esta prueba piloto se elaboró una propuesta de taller transdisciplinario que consistió en el diseño curricular de tres talleres con 15 sesiones de dos horas por período semestral; las sesiones se desarrollaron con exposiciones por parte de profesores y alumnos, con actividades de sensibilización, de investigación y de trabajo en equipo; se realizaron sesiones de análisis, reflexión y discusión de ideas a nivel grupal.

Para el diseño del taller se tomaron en cuenta las siguientes consideraciones: Utilizar los distintos tipos de lenguaje (escrito, de la imagen, gráfico, textual, gestual, artístico, virtual, matemático, articulado), las 
herramientas tecnológicas de información y comunicación, así como los recursos didácticos necesarios para lograr un estado de atención que permita acceder al nivel de aprendizaje significativo y fomentar el desarrollo integral de las habilidades de comunicación lingüística, de investigación y de comunicación de conocimientos científicos.

El diseño curricular fue sometido a una muestra intencional de 10 académicos, denominados expertos, quienes fueron seleccionados entre los profesores de educación superior de diferentes disciplinas de ingeniería de la UADY, con base en su alto nivel de competencias y experiencia profesional. Los expertos evaluaron individualmente el programa del taller mediante un instrumento que les fue proporcionado, dictaminando respecto del grado de cumplimiento de los requisitos estructurales de contenido que debe tener todo proyecto de innovación curricular. Los datos proporcionados por los expertos se procesaron a través del software estadístico SPSS y se les aplicó la prueba no paramétrica para una sola muestra de $\chi^{2}$ (Siegel y Castellan, 2002). El tipo de muestreo fue no probabilístico e intencional y la técnica utilizada para el análisis fue del tipo bondad de ajuste, para probar la existencia o no de una diferencia significativa entre el número de respuestas dadas por los expertos y el número de respuestas esperadas.

En la impartición de los talleres, denominados "Arte-Ciencia”, participaron dos profesoras de la Facultad de Ingeniería de la UADY, una especialista en antropología, lingüística y literatura, y otra con experiencia en la disciplina de física. Los tres talleres estuvieron dirigidos a un mismo grupo de 15 alumnos de la Facultad de Ingeniería de la UADY de las ingenierías civil, física y mecatrónica, que cursaban asignaturas diversas (tronco común) de los primeros dos semestres de las licenciaturas, que voluntariamente se incorporaron al mismo y participaron durante los tres semestres de duración. Se realizaron mediciones antes y después de haber puesto en práctica el taller. Para medir la influencia formativa del taller sobre la variable dependiente estudiada y el logro de los objetivos del mismo, se empleó un instrumento para evaluar el nivel de desarrollo de competencias comunicativas y de investigación en este campo, construido por Pérez y Sarlabous (2005), de acuerdo con las recomendaciones de Hernández, Fernández y Baptista (2006). Para las pruebas de hipótesis para las cinco variables dependientes (nivel de comprensión, nivel de aplicación, nivel de metarreflexión, nivel de comunicación y nivel de motivación) se empleó la prueba no paramétrica para dos grupos relacionados, de rangos señalados y pares igualados de Wilcoxon (Siegel y Castellán, 2002). Así mismo, al final de los talleres, se aplicó un cuestionario a los estudiantes con vista a conocer las valoraciones sobre los propósitos que persiguió el taller, participación de los cursantes en la realización de ajustes a la programación y desarrollo del taller, desempeño de los maestros facilitadores, calidad de los contenidos abordados, aprendizaje significativo de los contenidos temáticos abordados, calidad de las tareas y actividades realizadas en el curso y el contexto ambiental en que fue desarrollado el taller.

\section{RESULTADOS Y DISCUSIÓN}

El proyecto se propuso y desarrolló con el nombre de Taller Transdisciplinario de Competencias de Comunicación de Conocimientos Científicos y de Investigación, con un perfil de egreso de los participantes vinculado a las competencias siguientes:

Comunicarse asertivamente en el contexto en que se encuentre.

Solucionar problemas con responsabilidad social.

Interactuar con grupos heterogéneos, ejerciendo su autonomía de manera responsable.

Utilizar habilidades de investigación en el ejercicio profesional.

Desempeñarse eficazmente en equipo de trabajo transdisciplinario.

Debatir ideas y conceptos con respeto y tolerancia hacia diferentes corrientes de pensamiento.

Dominar los procedimientos organizacionales para la presentación de un proyecto de investigación.

Ejercer una ciudadanía responsable

Propósito clave: Los módulos de desarrollo de competencias de comunicación de conocimientos científicos, tienen como propósito clave, sensibilizar a los estudiantes para desarrollar de manera integral competencias de comunicación lingüística e investigación, que les permitan participar en interacción con sus compañeros y miembros externos al contexto educativo; así como desarrollar habilidades de dominio simbólico y tecnológico en el proceso de construcción de un proyecto de investigación.

Unidad de competencia (formativa): Interpretar en el proceso de la comunicación, los signos y símbolos de la comunicación verbal, no verbal y de tecnología, en interacción social en grupos heterogéneos, que permitan al estudiante ejercer su autonomía de manera responsable.

Para lograr este propósito, se realizarán actividades que permitan desarrollar las siguientes competencias genéricas: 
Competencias cognitivas: (interacción social en grupos heterogéneos)

Reafirmar la importancia del proceso de la comunicación en todos los aspectos y distintos contextos de la vida del individuo.

Desarrollar habilidades para expresarse de manera verbal, no verbal y el uso de tecnologías, de manera coherente y clara.

Aplicar técnicas de lectura de comprensión, de redacción, expresión e investigación

Desarrollo y actualización en las tecnologías de información y comunicación (TIC).

Competencias de orden ético y ciudadano: (autonomía)

Exponer los conocimientos adquiridos, con asertividad y fluidez.

Dominar los procedimientos organizacionales para la presentación del proyecto de investigación que haya elegido de acuerdo a su perfil.

Manifestar actitudes de integración social, a través del uso de herramientas de los diferentes tipos de lenguaje, para comunicarse de una manera clara, concisa y ética.

Emprender la colaboración en un equipo de trabajo transdisciplinario, con fines de investigación para la consolidación de un proyecto, dentro y fuera de la universidad.

Debatir ideas y conceptos, con respeto y tolerancia hacia las diferentes posturas ideológicas.

Conocer acerca de los derechos y obligaciones de los seres humanos, a fin de ejercer una ciudadanía responsable.

La orientación que brinda este taller, se hará teniendo en cuenta los diferentes aportes de los participantes y sus contactos en el proceso de investigación, prefiriéndose los enfoques críticos, que inviten a la reflexión y al análisis de las situaciones planteadas. Se organizará en torno a los siguientes ejes temáticos o módulos de conocimiento:

Unidades de aprendizaje:

Unidad 1: Condiciones para realizar una comunicación asertiva en distintos contextos. Fases: Despertar la percepción, ingresando al arte - ciencia, arte - ciencia y creación en expresión.

Unidad 2: El estudio de temas científicos y de otros, a través de la lectura para el desarrollo de habilidades de razonamiento y pensamiento crítico. Fases: Desarrollo de la percepción, desarrollo del arte - ciencia, arte - ciencia y creación en expresión.

Unidad 3: Interpretación de situaciones problema en temas de interés de las ingenierías, y comunicarlos estableciendo condiciones. Fases: Uso de la percepción, Expresando arte - ciencia, arte - ciencia y creación en expresión.

En las primeras tres fases de cada unidad se emplearon algunas técnicas de meditación y de movimiento corporal para lograr la relajación del cuerpo y de la mente; en la segunda fase de cada una de las unidades se incluyó la actividad de investigación, desde la selección del tema científico por investigar, relacionado con el perfil profesional, hasta la clasificación e integración de la información de una manera coherente, clara y concisa para efectos de comunicación, trabajando en equipo interdisciplinario para desarrollar los conocimientos. En la fase tres se elaboró el guión y se realizó la adaptación de manera creativa para la representación de los conceptos científicos interpretados por cada uno de los participantes, utilizando ellos sus competencias y habilidades desarrolladas en este contexto, empleando diversos tipos de lenguaje, así como recursos gráficos y de tecnología para describir la secuencia de escenas, personajes y ambientación. En la fase cuatro de cada módulo se desarrolló el trabajo multidisciplinariamente entre los participantes y especialistas contactados con el propósito de consolidar el proceso creativo y cognitivo, creando los efectos tecnológicos especializados audiovisuales para poder captar la atención respecto de sus propios actos, al momento de expresar los conocimientos científicos durante la representación escénica.

El conocimiento de estos contenidos, se construyó a través de las siguientes actividades de aprendizaje:

Lectura de investigación científica.

Redacción de trabajos de investigación científica.

Dinámicas.

Elaboración de guiones de entrevistas.

Entrevistas, en persona y por la Web.

Visitas a empresas de interés de acuerdo al perfil profesional.

Exposición de temas ante grupo presencial o virtual.

Exhibición de películas de acuerdo al quehacer profesional.

Realización de mapas geográficos, maquetas.

Práctica con software (programas o paquetes) de acuerdo al interés de la especialidad. 
Comunicación y expresión de conocimientos, utilizando como herramienta cualquier tipo de lenguaje.

Técnicas de escucha y de concentración.

Práctica en debates.

Observar la coherencia en las actividades comunicativas.

Interactuar en equipo transdisciplinario.

El tratamiento colectivo de los ejes temáticos, donde profesor/es y estudiante/s construirán los conocimientos propios del taller, propiciará la adquisición de otras competencias específicas de la comunicación de conocimientos científicos, cuya evaluación se hará teniendo en cuenta las principales subcompetencias indicadas en cada uno de los módulos.

Contenido de las unidades

Unidad 1: Condiciones para realizar una comunicación asertiva en distintos contextos. Esta unidad comprende la revisión de la concepción de la comunicación asertiva en los ámbitos educativo y social, y su relación con el autoconcepto; se espera que esta primera unidad permita la construcción de una base cognitiva lo suficientemente amplia y orientadora para facilitar el aprendizaje de las unidades siguientes.

Elemento de competencia:

Adoptar los principios de asertividad que permitan al discente interactuar adecuadamente en el contexto en el que se desenvuelve.

Unidad 2: La comprensión de lectura de temas científicos y otros.

Esta unidad comprende el estudio de temas científicos y de otros, a través de la lectura para el desarrollo de habilidades de razonamiento, pensamiento crítico y autónomo.

Elemento de competencia:

Reconocer la importancia de realizar lectura de temas científicos, con una visión abierta, buscando el desarrollo del pensamiento crítico y autónomo.

Unidad 3: Interpretación de situaciones-problema en temas de interés de las ingenierías, comunicarlos, estableciendo las condiciones en que se encuentren.

Esta unidad comprende la interacción de las principales fases del acto comunicativo, con sus diferentes formas de expresión o producción e interpretación.

Elemento de competencia:

Interpretar con responsabilidad social, los lenguajes verbal y no verbal, buscando comprender la vinculación que pueden tener los contenidos científicos con la vida cotidiana, y con las necesidades sociales que se presentan en la actualidad.

Para el primer taller se eligió por consenso como tema de investigación "La teoría de la relatividad" del científico Albert Einstein. Para el segundo, los participantes desarrollaron la escenificación de la parte medular de la teoría investigada en el primer taller, bajo la denominación concretizada en " $E=\mathrm{mc}^{2}$ ", presentando todos los conocimientos adquiridos en el proceso de investigación previo, utilizando un lenguaje científico pero entendible para el público asistente. Para el tercer taller, los participantes decidieron por consenso investigar acerca de "La teoría del caos", desarrollando y construyendo de manera autónoma el argumento teatral, basado en un pasaje de la obra "La fundación" de Isaac Asimov.

El instrumento utilizado para la evaluación de la propuesta curricular del taller por parte de los expertos, usó cuatro niveles de respuesta: muy bien, bien, regular y mal. Los resultados de la evaluación por parte de ellos aparecen en la tabla 1.

Como se puede observar en la evaluación de los expertos, tabla 1, únicamente en tres apartados (27\%) de la propuesta hubo una calificación otorgada en el nivel de regular, con la opinión de un experto en cada apartado; entre un $60 \%$ y un $90 \%$ de los expertos calificaron todos los rubros en la escala de muy bien; ocho apartados (73\%) fueron calificados en los niveles bien y muy bien. Los expertos precisaron que la finalidad de la propuesta del taller está adecuadamente planteada y que en la misma se precisan sus beneficiarios directos e indirectos, expresa con claridad los objetivos educativos que se propone lograr y que los mismos son posibles de alcanzar, traza las orientaciones metodológicas y la estrategia general que se debe seguir para el logro de los objetivos, expone los cursos generales de acción que se seguirán en el desarrollo del taller y los lineamientos que se deben cumplir para la puesta en práctica de su secuencia de actividades, así como que éstas se derivan y apoyan en la fundamentación del programa. 
Tabla 1. Resultados de la evaluación por parte de los expertos.

\begin{tabular}{|l|c|c|c|c|}
\hline \multirow{2}{*}{ Apartado } & Calificación (\%) & Calificación (\%) & Calificación (\%) & Calificación (\%) \\
\cline { 2 - 5 } & Muy Bien & Bien & Regular & Mal \\
\hline Presentación & 90 & 10 & & \\
\hline Introducción & 60 & 40 & & \\
\hline Fundamentación & 70 & 30 & & \\
\hline Finalidad y beneficiarios & 90 & 40 & & \\
\hline Objetivos generales & 60 & 40 & & \\
\hline $\begin{array}{l}\text { Orientaciones } \\
\text { metodológicas }\end{array}$ & 60 & 10 & & \\
\hline Unidades estructurales & 80 & 20 & & \\
\hline Objetivos específicos & 80 & & & \\
\hline Actividades particulares & 90 & 20 & & \\
\hline $\begin{array}{l}\text { Orden de sucesión de las } \\
\text { actividades }\end{array}$ & 80 & 20 & & \\
\hline $\begin{array}{l}\text { Tiempos de duración de } \\
\text { las actividades }\end{array}$ & 70 & & & \\
\hline
\end{tabular}

La aplicación de la prueba de $\chi^{2}$ a los resultados anteriormente presentados, arrojó que todos ellos son estadísticamente significativos al nivel de confianza 0.05. Por lo tanto, sí hubo diferencias en la opinión de los expertos con respecto al diseño de los talleres; sin embargo, todos los aspectos fueron valorados positivamente, debiéndose las diferencias a grados en los cuales consideraron bien o muy bien elaboradas las características antes mencionadas, siendo mínimo el número de expertos que opinó que algunos apartados del diseño propuesto era regular.

Las evaluaciones obtenidas a través de la administración del instrumento denominado "Escala de evaluación de las competencias comunicativas e investigativas del estudiante", antes y después del taller a los alumnos en las 5 variables dependientes analizadas (nivel de comprensión, nivel de aplicación, nivel de metarreflexión, nivel de comunicación y nivel de motivación), proporcionaron evidencias cuantitativas que mostraron la efectividad de la propuesta pedagógica para la innovación educativa. La prueba no paramétrica de Wilcoxon para rangos asignados, a un nivel de confianza de 0.01 , en las variables estudiadas, reportaron que los resultados obtenidos en las evaluaciones finales fueron muy superiores a los que se obtuvieron en las evaluaciones iniciales, es decir, las diferencias encontradas fueron altamente significativas para los 15 alumnos participantes. Al agrupar las 5 variables dependientes analizadas, se constató que los recuentos totales de las evaluaciones (Mal, Regular, Bien y Muy Bien), antes y después de realizado el taller, arrojan que los resultados obtenidos por los alumnos en sus evaluaciones finales, fueron similares a las variables individuales y muy superiores a los resultados obtenidos en las evaluaciones iniciales tal como puede observarse en la figura 1.

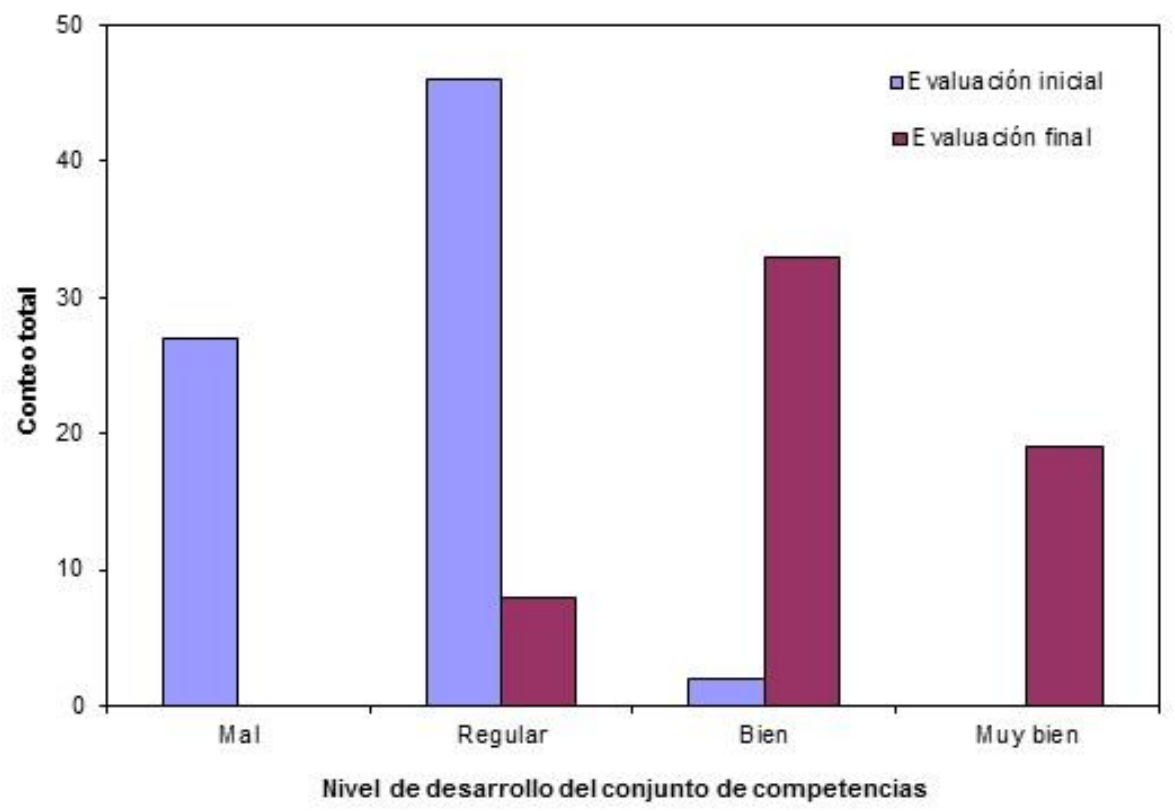

Fig. 1: Recuento total de las evaluaciones iniciales y finales del desarrollo global de las competencias en los alumnos estudiados. 
Respecto de las valoraciones finales de los estudiantes acerca del taller, el $100 \%$ de ellos consideró que los objetivos fueron relevantes para la adquisición de sus competencias comunicativas y de investigación, aceptando que tuvieron incidencia positiva para el mejoramiento de su proceso de aprendizaje en la licenciatura. Los alumnos participantes consideraron que la calidad general del desempeño de los profesores involucrados fue buena, que los contenidos presentaban nivel científico con posibilidades de aplicación práctica, y que las tareas y actividades de aprendizaje fueron adecuadas para la consecución de los objetivos del taller.

Participar en un taller transdisciplinario es una oportunidad para que los alumnos experimenten, reflexionen, analicen y se retroalimenten tanto con sus conocimientos formales como con los que adquieran a nivel extracurricular. Esto puede contribuir a elevar sus niveles de creatividad y a mejorar su relación con el entorno, enfocado a su quehacer científico y desempeño profesional, desarrollando habilidades socioafectivas tales como: el respeto a las personas, el ejercicio de una ciudadanía responsable, la honestidad, la empatía con quienes trabaja de manera cooperativa, y la expresión libre de ideas y sentimientos.

\section{CONCLUSIONES}

Se diseñó un taller transdisciplinario, con el propósito de elevar el nivel de competencias de comunicación lingüística y de investigación de los alumnos del Campus de Ciencias Exactas e Ingenierías de la Universidad Autónoma de Yucatán y se impartió a un grupo de 15 alumnos. Las evaluaciones de las competencias comunicativas e investigativas de los estudiantes antes y después de la impartición del taller, presentan evidencias cuantitativas que muestran la efectividad de la propuesta pedagógica implementada.

Los profesores de la Facultad de Ingeniería de la UADY, expertos en sus áreas disciplinares respectivas, que fueron consultados respecto de la propuesta del taller transdisciplinario, lo calificaron de forma positiva.

\section{REFERENCIAS}

Agudo, J., Hernández, R., Rico, M. y Sánchez H., Competencias transversales: Percepción de su desarrollo en el grado en ingeniería en diseño industrial y desarrollo de productos, Formación Universitaria, Vol. 6(5), 39-50 (2013).

Ander-Egg, E., El Taller. Una alternativa para la renovación pedagógica. Magisterio del Río de La Plata, Buenos Aires, Argentina (1991).

Ausubel, D., Psicología educativa. Un punto de vista cognitivo. Trillas, México (1990).

Bellocchio, M., Educación basada en competencia y constructivismo. ANUIES, México (2010).

Benítez, A. y García, M. Un primer acercamiento al docente frente a una metodología basada en proyectos. Formación Universitaria. Vol. 6(1), 21-28 (2013).

Canales, T. y Schmal, R., Trabajando con pósteres: una Herramienta para el desarrollo de habilidades de comunicación en la educación de pregrado. Formación Universitaria, Vol. 6(1), 41-52 (2013).

Carlino, P., Alfabetización académica: un cambio necesario, algunas alternativas posibles. 1aㅡ edición, 411, Educere, año 6, No. 20 Enero-Febrero-Marzo (2003).

Castellanos, B., La formación de la competencia investigativa: una necesidad y una oportunidad para mejorar la calidad de la educación. Memorias Congreso Pedagogía 2003, La Habana, Cuba (2003).

Castro, C., Chapato, M. y Troiano M., El desarrollo de competencias de comunicación oral y escrita. Experiencia con alumnos de la carrera de profesorado de teatro. 1 Congreso internacional "Educación, lenguaje y sociedad", La Pampa, Argentina (2004).

Hernández, S., Fernández, C. y Baptista, M., Metodología de la Investigación. 5aㅗ edición, McGraw-Hill Interamericana. Perú (2006).

Hymes, D., Acerca de la competencia comunicativa, Revista Forma y Función, Junio 1996. Santa Fe, Bogotá. (1972).

Maldonado, M., Las competencias, una opción de vida: metodología para el diseño curricular. ECOE, Bogotá (2005). 
Martínez, F., ¿Es posible una formación sistemática para la investigación educativa? Algunas reflexiones. Revista electrónica de Investigación Educativa, Vol. 1(1),1-6 (1999).

Mittendorf, I. y Cox, J.,Around the $\beta$-Turn: An Activity To Improve the Communication and Listening Skills of Biochemistry Students. Journal of Chemical Education, Vol. 90 (11), 1476-1478 (2013).

Nicolescu, B., Manifesto of Transdisciplinarity. State University of New York (SUNY) Press, Nueva York, USA (2002).

Pérez, G. y Sarlabous, A., Escala de Evaluación de Competencias Investigativas y Comunicativas de los Alumnos. Pueblo y Educación, La Habana, Cuba (2005).

Piaget, J., Educación e instrucción. Proteo, Buenos Aires, Argentina (2005).

Piaget, J., Psicología y pedagogía. Ariel, Barcelona, España (2006).

Piaget, J., Seis Estudios de Psicología. Seix Barra, Barcelona, España (2008).

Polaco, A. y Manjarrez, L., La necesidad de la integración multidisciplinaria e interdisciplinaria en la educación. Recuperado en: http://fch.mxl.uabc.mx/lateduca/219.pdf (2010).

Regalado, A., Peralta E. y Báez G., Aprendizaje basado en competencias aplicado a una asignatura de transferencia de calor, Formación Universitaria, Vol. 4(1), 13-18 (2011).

Rodríguez, M., Mena, D. y Rubio, C., Competencias que pueden desarrollarse en la asignatura de mecánica en el currículo de ingeniería, Formación Universitaria, Vol. 4(4), 3-12 (2011).

Ruiz, M., El concepto de competencias desde la complejidad: hacia la construcción de competencias educativas. Trillas, México (2010).

Siegel, S. y Castellan, J., Estadística no Paramétrica. Aplicada a las Ciencias de la Conducta. Trillas, México (2002).

Squier, C., Renaud, J. y Larsen S., Integration of a Communicating Science Module into an Advanced Chemistry Laboratory Course. Journal of Chemical Education, Vol. 83 (7), 1029 (2006).

Tobón, S., Formación basada en competencias. Pensamiento complejo, diseño curricular y didáctica. ECOE, Bogotá, Colombia (2008).

Tobón, S., Pimienta, J. y García, F., Secuencias didácticas: aprendizaje y evaluación de competencias. Pearson, México (2010).

UADY, Modelo Educativo para la Formación Integral (MEFI). Plan de Desarrollo institucional 2010-2020. Recuperado en: http://www.pdi.uady.mx/mefi/contenido.php (2010).

Vigotsky, L., El desarrollo de los procesos psicológicos superiores. Crítica, Barcelona, España (1994).

Vigotsky, L., Pensamiento y Lenguaje. Ediciones Revolucionarias, Instituto del Libro, La Habana, Cuba (1996).

Werner, T., Reflections on the emphasis of communication skills in the undergraduate chemistry curriculum. Journal of Chemical Education, Vol. 63 (2), 140 (1986). 